\title{
Infinitesimal initial part of a singular foliation
}

\author{
NURIA CORRAL \\ Departamento de Matemáticas, Estadística y Computación, Universidad de Cantabria \\ Avda. de los Castros s/n, 39005 Santander, Spain \\ Manuscript received on August 29, 2008; accepted for publication on April 4, 2009; \\ presented by ARON SIMIS
}

\begin{abstract}
This work provides a necessary and sufficient condition to assure that two generalized curve singular foliations have the same reduction of singularities and same Camacho-Sad indices at each infinitely near point.
\end{abstract}

Key words: singular holomorphic foliations, complex dynamics.

\section{INTRODUCTION}

The germs of holomorphic foliations over $\left(\mathbb{C}^{2}, 0\right)$ are dynamical objects much more general than curves or even levels of meromorphic functions. In (Camacho et al. 1984), Camacho, Lins Neto and Sad introduce a class of foliations that share the reduction of singularities with their curve of separatrices. A more accurate approximation to a foliation (that assures coherent linear holonomies) is to compare them with levels of multivalued functions (logarithmic foliations); this has been done in (Corral 2003) by adding a control of the Camacho-Sad indices. In this paper we show that the necessary and sufficient condition for two foliations to share reduction of singularities, separatrices and Camacho-Sad indices is to have the same initial parts up to blow-up.

Let $M$ be an analytic complex manifold of dimension two and consider two germs of foliations $\mathcal{F}$ and $\mathcal{G}$ defined in a neighbourhood of a point $p \in M$. Let $\omega^{\mathcal{F}}, \omega^{\mathcal{G}} \in \Omega_{M, p}^{1}$ be 1 -forms defining $\mathcal{F}$ and $\mathcal{G}$ respectively in a neighbourhood of $p$. It is clear that if the $n$-jets of $\omega^{\mathcal{F}}$ and $\omega^{\mathcal{G}}$ coincide for $n$ big enough, then the foliations $\mathcal{F}$ and $\mathcal{G}$ will share all the properties mentioned above. But this condition is not necessary as we show with examples.

\section{LOCAL INVARIANTS}

Let $M$ be an analytic complex manifold of dimension two and let $\mathcal{F}$ be a singular holomorphic foliation on $M$. In a neighbourhood of any point $p \in M$, the foliation $\mathcal{F}$ is defined by a 1 -form

$$
\omega=A d x+B d y
$$

E-mail: nuria.corral@unican.es 
with $A, B \in \mathcal{O}_{M, p}$ and $\operatorname{gcd}(A, B)=1$. The multiplicity $v_{p}(\mathcal{F})$ of $\mathcal{F}$ at $p$ is the minimum of the multiplicities $v_{p}(A), v_{p}(B)$ (note that $v_{p}()$ is the vanishing order at $p$ ). The point $p$ is called a singular point of $\mathcal{F}$ if $v_{p}(\mathcal{F}) \geq 1$.

Let $S$ be a germ of an irreducible analytic curve at $p$. We say that $S$ is a separatrix of $\mathcal{F}$ through $p$ if $f$ divides $\omega \wedge d f$, where $f=0$ is a reduced equation of $S$. Denote by $\operatorname{Sep}_{p}(\mathcal{F})$ the set of separatrices of $\mathcal{F}$ through $p$.

Let $\pi_{1}: M_{1} \rightarrow(M, p)$ be the blow-up of $M$ with center at $p$ and let $E_{1}$ be the exceptional divisor $\pi_{1}^{-1}(p)$. The blow-up $\pi_{1}$ is called non-dicritical if $E_{1}$ is invariant by the strict transform $\pi_{1}^{*} \mathcal{F}$ of $\mathcal{F}$ by $\pi_{1}$; otherwise, $E_{1}$ is generically transversal to $\pi_{1}^{*} \mathcal{F}$ and we say that $\pi_{1}$ is dicritical.

Consider now a non-singular invariant curve $S=(y=0)$ of the foliation $\mathcal{F}$ through $p$. The multiplicity of $\mathcal{F}$ at $p$ along $S$ is given by

$$
\mu_{p}(\mathcal{F}, S)=\operatorname{ord}_{x}(B(x, 0))
$$

(see (Camacho et al. 1984)). Notice that it coincides with the multiplicity of the restriction $\left.\xi\right|_{S}=$ $B(x, 0) \partial / \partial x$ of the vector field $\xi=B(x, y) \partial / \partial x-A(x, y) \partial / \partial y$ to the curve $S$. Its behaviour under blow-up is given by

$$
\begin{array}{ll}
\mu_{p_{1}}\left(\pi_{1}^{*} \mathcal{F}, S_{1}\right)=\mu_{p}(\mathcal{F}, S)-v_{p}(\mathcal{F})+1 & \text { if } \pi_{1} \text { is non-dicritical, } \\
\mu_{p_{1}}\left(\pi_{1}^{*} \mathcal{F}, S_{1}\right)=\mu_{p}(\mathcal{F}, S)-v_{p}(\mathcal{F}) & \text { if } \pi_{1} \text { is dicritical, }
\end{array}
$$

where $S_{1}$ is the strict transform of $S$ by $\pi_{1}$ and $p_{1}=S_{1} \cap E_{1}$. Moreover, we have that

$$
\sum_{q \in E_{1}} \mu_{q}\left(\pi_{1}^{*} \mathcal{F}, E_{1}\right)=v_{p}(\mathcal{F})+1
$$

The Camacho-Sad index $I_{p}(\mathcal{F}, S)$ of $\mathcal{F}$ relative to $S$ at $\mathrm{p}$ is given by

$$
I_{p}(\mathcal{F}, S)=-\operatorname{Res}_{x=0} \frac{a(x, 0)}{B(x, 0)}
$$

where $A(x, y)=y a(x, y)$ (see (Camacho and Sad 1982)).

It is important to notice that, if $\omega, \omega^{\prime} \in \Omega_{M, p}^{1}$ are 1-forms defining $\mathcal{F}$ in a neighbourhood of $p$, then $\omega=h \cdot \omega^{\prime}$ where $h \in \mathcal{O}_{M, p}$ is a unit. In particular, this implies that the jets of order $v_{p}=v_{p}(\mathcal{F})$ of $\omega$ and $\omega^{\prime}$ coincide up to multiplication by a constant. We can write $\omega=\sum_{j \geq v_{p}} \omega_{j}$, where the coefficients of $\omega_{j}$ are homogeneous polynomials of degree $j$. Then the projective class $\left[\omega_{v_{p}}\right]$ of the $v_{p}$-jet of $\omega$ is well defined. We call $J_{p}(\mathcal{F})=\left[\omega_{v_{p}}\right]$ the initial part of $\mathcal{F}$ at $p$.

Let us write $\omega_{j}=A_{j} d x+B_{j} d y$ with $A_{j}, B_{j} \in \mathcal{O}_{M, p}$ and consider the homogeneous polynomial of degree $v_{p}+1$ given by $P_{v_{p}+1}=x A_{v_{p}}+y B_{v_{p}}$. The tangent cone $T C_{p}(\mathcal{F})$ of $\mathcal{F}$ at $p$ is given by

$$
P_{v_{p}+1}(x, y)=0 \text {. }
$$

If $P_{v_{p}+1} \not \equiv 0$, then $T C_{p}(\mathcal{F})$ is a finite union of lines and we denote by $\mathfrak{p}_{p}(\mathcal{F})$ the projective class of the polynomial $P_{v_{p}+1}$. Notice that two foliations with different initial parts, may have the same tangent cone. Moreover, the blow-up $\pi_{1}$ is non-dicritical if and only if $P_{v_{p}+1} \not \equiv 0$. 
Let us now recall the construction of the Newton polygon of a foliation. Taking local coordinates $(x, y)$ in a neighbourhood of $p \in M$, a germ $f \in \mathcal{O}_{M, p}$ of a function at $p$ can be written as a convergent power series $f=\sum_{i+j \geq v_{p}(f)} f_{i j} x^{i} y^{j}$. We denote $\Delta(f ; x, y)=\left\{(i, j): f_{i j} \neq 0\right\}$. The Newton polygon $\mathcal{N}(f ; x, y)$ is the convex hull of $\Delta(f ; x, y)+\left(\mathbb{R}_{\geq 0}\right)^{2}$. If $C$ is the germ of curve at $p$ defined by $f=0$, then $\mathcal{N}(C ; x, y)=\mathcal{N}(f ; x, y)$. Consider now a germ of foliation $\mathcal{F}$ given by $\omega=A(x, y) d x+B(x, y) d y$ in a neighbourhood of $p$. The Newton polygon $\mathcal{N}(\mathcal{F} ; x, y)$ of $\mathcal{F}$ is the convex hull of $\Delta(\omega ; x, y)+\left(\mathbb{R}_{\geq 0}\right)^{2}$ where $\Delta(\omega ; x, y)=\Delta(x A ; x, y) \cup \Delta(y B ; x, y)$.

Finally, let us recall the desingularization process of a foliation. We say that $p$ is a simple singularity of $\mathcal{F}$ if there are coordinates $(x, y)$ centered at $p$ so that $\mathcal{F}$ is defined by a 1 -form of the type

$$
\lambda y d x-\mu x d y+\text { h.o.t. }
$$

with $\mu \neq 0$ and $\lambda / \mu \notin \mathbb{Q}_{>0}$. If $\lambda=0$, the singularity is called a saddle-node. A reduction of singularities of $\mathcal{F}$ is a morphism

$$
\pi: M^{\prime} \rightarrow(M, p)
$$

composition of a finite number of blow-ups of points such that the strict transform $\pi^{*} \mathcal{F}$ of $\mathcal{F}$ by $\pi$ satisfies that

- each irreducible component of the exceptional divisor $D=\pi^{-1}(p)$ is either invariant by $\pi^{*} \mathcal{F}$ or transversal to $\pi^{*} \mathcal{F}$;

- all the singular points of $\pi^{*} \mathcal{F}$ are simple and do not belong to a dicritical component of the exceptional divisor $D$.

The minimal morphism $\pi_{\mathcal{F}}$, in the sense that it cannot be factorized by another morphism with the above properties, is called the minimal reduction of singularities of $\mathcal{F}$. The centers of the blow-ups of any reduction of singularities of $\mathcal{F}$ are called infinitely near points of $\mathcal{F}$. In particular, all the centers of the blow-ups to obtain $\pi_{\mathcal{F}}$ and the final singularities in $\pi_{\mathcal{F}}^{-1}(p)$ are infinitely near points of $\mathcal{F}$. This notion extends the well known notion of infinitely near points of a curve.

\section{GENERALIZED CURVE FOLIATIONS}

Let $\mathbb{F}=\mathbb{F}(M, p)$ be the space of singular holomorphic foliations of $(M, p)$. We denote by $\mathbb{G}$ the subspace of $\mathbb{F}$ composed by generalized curve foliations, that is, foliations without saddle-node singularities in their reduction of singularities (see (Camacho et al. 1984)). Given a germ $C=\cup_{i=1}^{r} C_{i}$ of analytic curve in $(M, p)$, we denote by $\mathbb{F}_{C}$ the subspace of $\mathbb{F}$ composed by foliations whose curve of separatrices is $C$, and we put $\mathbb{G}_{C}=\mathbb{G} \cap \mathbb{F}_{C}$. Notice that foliations in $\mathbb{F}_{C}$ are non-dicritical. Particular elements of $\mathbb{G}_{C}$ are the foliations given by $d f=0$, where $f=0$ is a reduced equation of $C$; let $\mathcal{G}_{C}$ be one of such foliations. It is known (see (Camacho et al. 1984, Rouillé 1999)) that, for an element $\mathcal{F} \in \mathbb{G}_{C}$, we have that:

(i) The minimal reduction of singularities of $\mathcal{F}$ and $C$ coincide.

(ii) The multiplicities satisfy $v_{p}(\mathcal{F})=v_{p}\left(\mathcal{G}_{C}\right)=v_{p}(C)-1$, where $v_{p}(C)=v_{p}(f)$. 
(iii) For any local coordinates $(x, y)$ in a neighbourhood of $p$, the Newton polygons $\mathcal{N}(\mathcal{F} ; x, y)$ and $\mathcal{N}(C ; x, y)$ coincide.

Moreover, we have the following result

LEMMA 1. Let $\pi:(\tilde{M}, \tilde{p}) \rightarrow(M, p)$ be a morphism composition of a finite number of punctual blowups. Let $D=\pi^{-1}(p)$ be the exceptional divisor and take $E \subset D$ an irreducible component with $\tilde{p} \in E$. Then, for any foliation $\mathcal{F} \in \mathbb{G}_{C}$, the strict transforms $\pi^{*} \mathcal{F}$ and $\pi^{*} \mathcal{G}_{C}$ satisfy the following

1. $v_{\tilde{p}}\left(\pi^{*} \mathcal{F}\right)=v_{\tilde{p}}\left(\pi^{*} \mathcal{G}_{C}\right)$;

2. If $(\tilde{x}, \tilde{y})$ are local coordinates at $\tilde{p}$, then $\mathcal{N}\left(\pi^{*} \mathcal{F} ; \tilde{x}, \tilde{y}\right)=\mathcal{N}\left(\pi^{*} \mathcal{G}_{C} ; \tilde{x}, \tilde{y}\right)$;

3. $\mu_{\tilde{p}}\left(\pi^{*} \mathcal{F}, E\right)=\mu_{\tilde{p}}\left(\pi^{*} \mathcal{G}_{C}, E\right)$.

REMARK 1. If $\pi_{1}: M_{1} \rightarrow(M, p)$ is the blow-up of $p$ with $E_{1}=\pi_{1}^{-1}(p)$ and $\pi_{1}^{*} C$ denotes the strict transform of $C$ by $\pi_{1}$, then $\mu_{q}\left(\pi^{*} \mathcal{G}_{C}, E\right)=\left(\pi^{*} C \cdot E\right)_{q}$ for any $q \in E_{1}$, where $(\cdot)_{q}$ denotes the intersection multiplicity at $q$.

Proof of Lemma 1. Assertions 1 and 2 are a direct consequence of the above properties (ii) and (iii) applied at each infinitely near point of $\mathcal{F}$. Let us prove assertion 3.

Let $(x, y)$ be coordinates in a neighbourhood of $p$ and take a 1-form $\omega=A(x, y) d x+B(x, y) d y$ defining $\mathcal{F}$ in a neighbourhood of $p$. Consider a reduced equation $f=0$ of $C$ and let $\mathcal{G}_{C}$ be the foliation defined by $d f=0$. If we denote $v=v_{0}(\mathcal{F})$, then the multiplicity $v_{p}(C)$ of $C$ at $p$ is equal to $v+1$. Therefore, we can write $f=\sum_{i \geq v+1} f_{i}, A=\sum_{i \geq v} A_{i}$ and $B=\sum_{i \geq v} B_{i}$ with $f_{i}, A_{i}$ and $B_{i}$ being homogeneous polynomials of degree $i$.

Consider $\pi_{1}: M_{1} \rightarrow(M, p)$ the blow-up of $p$ and denote by $E_{1}=\pi_{1}^{-1}(p)$ the exceptional divisor. Let us prove that

$$
\mu_{q}\left(\pi_{1}^{*} \mathcal{F}, E_{1}\right)=\mu_{q}\left(\pi_{1}^{*} \mathcal{G}_{C}, E_{1}\right)
$$

at each point $q \in E_{1}$. Then the result follows using similar arguments.

If $q \in E_{1}$ is a non-singular point of $\pi_{1}^{*} \mathcal{F}$, then $\mu_{q}\left(\pi_{1}^{*} \mathcal{F}, E_{1}\right)=0$. Since the singular points of $\pi_{1}^{*} \mathcal{F}$ and $\pi_{1}^{*} \mathcal{G}_{C}$ in $E_{1}$ coincide, we also have that $\mu_{q}\left(\pi_{1}^{*} \mathcal{G}_{C}, E_{1}\right)=0$. Consequently, we only have to prove equality (3) at the singular points $q_{1}, q_{2}, \ldots, q_{t}$ of $\pi_{1}^{*} \mathcal{F}$ in $E_{1}$. We can assume that all the singular points belong to the first chart of $E_{1}$ and we take coordinates $\left(x^{\prime}, y^{\prime}\right)$ with $E_{1}=\left(x^{\prime}=0\right)$ and $\pi_{1}\left(x^{\prime}, y^{\prime}\right)=\left(x^{\prime}, x^{\prime} y^{\prime}\right)$. In these coordinates, the foliation $\pi_{1}^{*} \mathcal{G}_{C}$ is given by

$$
\left((v+1) f_{v+1}\left(1, y^{\prime}\right)+x^{\prime}(\ldots)\right) d x^{\prime}+x^{\prime}\left(\frac{\partial f_{v+1}}{\partial y^{\prime}}\left(1, y^{\prime}\right)+x^{\prime}(\ldots)\right) d y^{\prime}=0 .
$$

Let us write $f_{v+1}(1, y)=k \cdot \prod_{l=1}^{t}\left(y-a_{l}\right)^{r_{l}}$ with $k \in \mathbb{C}^{*}$. We can assume that the point $q_{l}$ is given by $\left(0, a_{l}\right)$ in the coordinates $\left(x^{\prime}, y^{\prime}\right)$, for $l=1, \ldots, t$. In particular, we have that $\mu_{q_{l}}\left(\pi_{1}^{*} \mathcal{G}_{C}, E_{1}\right)=r_{l}$.

In the coordinates $\left(x^{\prime}, y^{\prime}\right)$, the foliation $\pi_{1}^{*} \mathcal{F}$ is given by $\omega_{1}=\pi_{1}^{*} \omega / x^{\prime \nu}=0$ with

$$
\omega_{1}=\left(P_{v+1}\left(1, y^{\prime}\right)+x^{\prime}(\cdots)\right) d x^{\prime}+x^{\prime}\left(B_{v}\left(1, y^{\prime}\right)+x^{\prime}(\cdots)\right) d y^{\prime} .
$$


By definition, we have that $\mu_{q_{l}}\left(\pi_{1}^{*} \mathcal{F}, E_{1}\right)=\operatorname{ord}_{y=a_{l}}\left(P_{v+1}(1, y)\right)$.

Take $\left(x_{l}, y_{l}\right)$ coordinates centered at $q_{l}$ with $x_{l}=x^{\prime}$ and $y_{l}=y^{\prime}-a_{l}$. From expression (4), we deduce that

$$
\mathcal{N}\left(\pi_{1}^{*} \mathcal{G}_{C} ; x_{l}, y_{l}\right) \subset\{(i, j): i \geq 1\}
$$

and $\mathcal{N}\left(\pi_{1}^{*} \mathcal{G}_{C} ; x_{l}, y_{l}\right) \cap\{i=1\}=\left\{(1, j): j \geq r_{l}\right\}$.

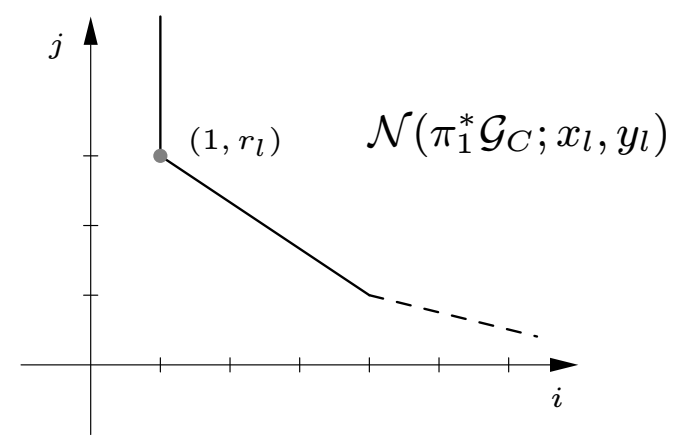

Then the equality of the Newton polygons $\mathcal{N}\left(\pi_{1}^{*} \mathcal{G}_{C} ; x_{l}, y_{l}\right)$ and $\mathcal{N}\left(\pi_{1}^{*} \mathcal{F} ; x_{l}, y_{l}\right)$ implies that the point $\left(1, r_{l}\right)$ belongs to $\Delta\left(\omega_{1} ; x_{l}, y_{l}\right)$. Taking into account that

$$
\Delta\left(\omega_{1} ; x_{l}, y_{l}\right) \cap\{i=1\}=\Delta\left(x_{l} P_{v+1}\left(1, y_{l}+a_{l}\right) ; x_{l}, y_{l}\right) \cup \Delta\left(x_{l} y_{l} B_{v}\left(1, y_{l}+a_{l}\right) ; x_{l}, y_{l}\right)
$$

we deduce that

$$
\operatorname{ord}_{y=a_{l}}\left(P_{v+1}(1, y)\right) \geq r_{l} ; \quad \operatorname{ord}_{y=a_{l}}\left(B_{v}(1, y)\right) \geq r_{l}-1 .
$$

Thus $\mu_{q_{l}}\left(\pi_{1}^{*} \mathcal{F}, E_{1}\right) \geq \mu_{q_{l}}\left(\pi_{1}^{*} \mathcal{G}_{C}, E_{1}\right)$ for each $l=1, \ldots, t$, and the following equality

$$
v+1=\sum_{l=1}^{t} \mu_{q_{l}}\left(\pi_{1}^{*} \mathcal{F}, E_{1}\right)=\sum_{l=1}^{t} \mu_{q_{l}}\left(\pi_{1}^{*} \mathcal{G}_{C}, E_{1}\right) .
$$

gives the result.

Corollary 1. Given two foliations $\mathcal{F}$ and $\mathcal{G}$ of $\mathbb{G}_{C}$, the polynomials $\mathfrak{p}_{p}(\mathcal{F})$ and $\mathfrak{p}_{p}(\mathcal{G})$ coincide at each infinitely near point $p$ of $C$.

\section{LOGARITHMIC FOLIATIONS}

Given a germ of plane curve $C=\cup_{i=1}^{r} C_{i}$ in $(M, p)$, a logarithmic foliation $\mathcal{L}$ in $\mathbb{G}_{C}$ is given by a closed meromorphic 1-form $\eta=0$ where

$$
\eta=\sum_{i=1}^{r} \lambda_{i} \frac{d f_{i}}{f_{i}}
$$

with $f_{i}=0$ being a reduced equation of $C_{i}$ and $\lambda_{i} \neq 0$. Notice that if $\eta$ as above defines a logarithmic foliation in $\mathbb{G}_{C}$, then the foliation given by $\eta+\alpha=0$, with $\alpha$ holomorphic and $d \alpha=0$, is also logarithmic and belongs to $\mathbb{G}_{C}$. In fact, we can write $\eta+\alpha=\sum_{i=1}^{r} \lambda_{i} d f_{i}^{*} / f_{i}^{*}$ for suitable equations $f_{i}^{*}=0$ of $C_{i}$. Thus, logarithmic foliations in $\mathbb{G}_{C}$ are characterized by their exponent vector $\underline{\lambda}=\left(\lambda_{1}, \ldots, \lambda_{r}\right) \in \mathbb{P}_{\mathbb{C}}^{r-1}$. 
We denote by $\mathcal{L}_{\underline{\lambda}}$ one of these foliations. Observe that not all the foliations defined by a 1 -form of the type (*) belong to $\mathbb{G}_{C}$ since they could be dicritical foliations.

Recall that a logarithmic foliation $\mathcal{L}$ is a logarithmic model of a foliation $\mathcal{F}$ if they have the same separatrices, the same reduction of singularities and the same Camacho-Sad indices at the final singularities after desingularization. The existence of logarithmic models for non-dicritical generalized curves was proved in (Corral 2003) and is unique once a reduced equation of the separatrices is fixed. Then, for each foliation $\mathcal{F} \in \mathbb{G}_{C}$, we denote by $\underline{\lambda}(\mathcal{F})$ the exponent vector of the logarithmic model of $\mathcal{F}$. We denote by $\mathbb{G}_{C, \underline{\lambda}}$ the set of foliations $\mathcal{F} \in \mathbb{G}_{C}$ such that $\underline{\lambda}(\mathcal{F})=\underline{\lambda}$. Thus, the set $\mathbb{G}_{C}$ is equal to

$$
\mathbb{G}_{C}=\cup_{\underline{\lambda} \in \mathbb{P}_{\mathbb{C}}^{r-1}} \mathbb{G}_{C, \underline{\lambda}} .
$$

Notice that $\mathbb{G}_{C, \underline{\lambda}} \neq \varnothing$ if and only if the foliation given by $\sum_{i=1}^{r} \lambda_{i} d f_{i} / f_{i}=0$ is non-dicritical. The goal of this article is to study the properties which characterize the foliations in one of such sets $\mathbb{G}_{C, \lambda}$.

Consider now a meromorphic 1 -form $\eta$ of the type $(*)$ and assume that the foliation given by $\eta=0$ belongs to $\mathbb{G}_{C}$. Thus all the foliations defined by $\eta+\alpha=0$, with $\alpha$ holomorphic, belong to $\mathbb{G}_{C, \underline{\lambda}}$ but in general they are not logarithmic. However, it is not true that all foliations of $\mathbb{G}_{C, \underline{\lambda}}$ are defined by a 1-form of the type $\eta+\alpha=0$ with $\alpha$ holomorphic.

\section{INFINITESIMAL INITIAL PART}

Given a non-dicritical foliation $\mathcal{F} \in \mathbb{F}$, we define the infinitesimal initial part $\mathcal{J}(\mathcal{F})$ of $\mathcal{F}$ to be the family $\left\{J_{q}(\mathcal{F})\right\}$ where $q$ varies among the infinitely near points of $\mathcal{F}$. We wonder under what conditions two foliations $\mathcal{F}$ and $\mathcal{G}$ have the same infinitesimal initial part. It is clear that having the same curve of separatrices and the same reduction of singularities are necessary conditions. But these conditions are not enough even if we work with generalized curve foliations. Notice also that a sufficient condition is that of $\mathcal{F}$ and $\mathcal{G}$ having the same $n$-jet at the point $p$, with $n$ being big enough. However, this condition is not necessary as shown by $d\left(y^{3}-x^{11}\right)=0$ and $11\left(-x^{10}+y x^{7}\right) d x+3\left(y^{2}-x^{8}\right) d y=0$. Then, the result is

THEOREM 1. Let $\mathcal{F}$ and $\mathcal{G}$ be two foliations in $\mathbb{G}_{C}$. The foliations $\mathcal{F}$ and $\mathcal{G}$ have the same infinitesimal initial part if and only if $\underline{\lambda}(\mathcal{F})=\underline{\lambda}(\mathcal{G})$.

Proof. Take $\mathcal{F}$ and $\mathcal{G}$ two foliations in $\mathbb{G}_{C}$ and assume that $\mathcal{J}(\mathcal{F})=\mathcal{J}(\mathcal{G})$. Consider the minimal reduction of singularities $\pi_{C}: \widetilde{M} \rightarrow(M, p)$ of $C$ (notice that $\pi_{C}=\pi_{\mathcal{F}}=\pi_{\mathcal{G}}$ ). By hypothesis, the initial parts $J_{q}(\mathcal{F})$ and $J_{q}(\mathcal{G})$ coincide at each point $q \in \pi_{C}^{-1}(p)$. In particular, this implies that the Camacho-Sad indices $I_{q}\left(\pi^{*} \mathcal{F}, F\right)$ and $I_{q}\left(\pi^{*} \mathcal{G}, F\right)$ coincide for a component $F \subset \pi_{C}^{-1}(p)$ through $q$ and consequently $\underline{\lambda}(\mathcal{F})=\underline{\lambda}(\mathcal{G})$.

Reciprocally, take a foliation $\mathcal{F} \in \mathbb{G}_{C, \underline{\lambda}}$ and let us show that $\jmath_{p}(\mathcal{F})=J_{p}(\mathcal{L})$, where $\mathcal{L}=\mathcal{L}_{\underline{\lambda}}$ is a logarithmic foliation in $\mathbb{G}_{C, \underline{\lambda}}$. Put $v=v_{p}(\mathcal{F})$ and assume that $\mathcal{F}$ and $\mathcal{L}$ are given by the holomorphic 1 -forms $\omega^{\mathcal{F}}=0$ and $\omega^{\mathcal{L}}=0$ respectively, where

$$
\begin{aligned}
& \omega^{\mathcal{F}}=\left(A_{v}^{\mathcal{F}}(x, y)+A_{v+1}^{\mathcal{F}}(x, y)+\cdots\right) d x+\left(B_{v}^{\mathcal{F}}(x, y)+B_{v+1}^{\mathcal{F}}(x, y)+\cdots\right) d y \\
& \omega^{\mathcal{L}}=\left(A_{v}^{\mathcal{L}}(x, y)+A_{v+1}^{\mathcal{L}}(x, y)+\cdots\right) d x+\left(B_{v}^{\mathcal{L}}(x, y)+B_{v+1}^{\mathcal{L}}(x, y)+\cdots\right) d y,
\end{aligned}
$$


with $A_{i}^{-}, B_{i}^{-}$being homogeneous polynomials of degree $i$. Consider the blow-up $\pi_{1}: M_{1} \rightarrow(M, p)$ of $p$ and let $q_{1}, q_{2}, \ldots, q_{t}$ be the singular points of $\pi_{1}^{*} \mathcal{F}$ in $E_{1}=\pi_{1}^{-1}(p)$. We can assume, without loss of generality, that all the points $q_{i}$ belong to the first chart of $E_{1}$. Take $\left(x^{\prime}, y^{\prime}\right)$ coordinates in the first chart of $E_{1}$ with $\pi_{1}\left(x^{\prime}, y^{\prime}\right)=\left(x^{\prime}, x^{\prime} y^{\prime}\right)$ and $E_{1}=\left(x^{\prime}=0\right)$. Then, the foliations $\pi_{1}^{*} \mathcal{F}$ and $\pi_{1}^{*} \mathcal{L}$ are given by $\omega_{1}^{\mathcal{F}}=0$ and $\omega_{1}^{\mathcal{L}}=0$ respectively, where

$$
\begin{aligned}
& \omega_{1}^{\mathcal{F}}=\left(P_{v+1}^{\mathcal{F}}\left(1, y^{\prime}\right)+x^{\prime}(\cdots)\right) d x^{\prime}+x^{\prime}\left(B_{v}^{\mathcal{F}}\left(1, y^{\prime}\right)+x^{\prime}(\cdots)\right) d y^{\prime} \\
& \omega_{1}^{\mathcal{L}}=\left(P_{v+1}^{\mathcal{L}}\left(1, y^{\prime}\right)+x^{\prime}(\cdots)\right) d x^{\prime}+x^{\prime}\left(B_{v}^{\mathcal{L}}\left(1, y^{\prime}\right)+x^{\prime}(\cdots)\right) d y^{\prime} .
\end{aligned}
$$

Assume that the point $q_{i}$ is given by $\left(0, a_{i}\right)$ in the coordinates $\left(x^{\prime}, y^{\prime}\right)$ and put $r_{i}=\mu_{q_{i}}\left(\pi_{1}^{*} \mathcal{F}, E_{1}\right)$. Thus, by Lemma 1 and equation (5), we have that

$$
P_{v+1}^{\mathcal{F}}(1, y)=k_{1} \prod_{i=1}^{t}\left(y-a_{i}\right)^{r_{i}} ; \quad P_{v+1}^{\mathcal{L}}(1, y)=k_{2} \prod_{i=1}^{t}\left(y-a_{i}\right)^{r_{i}},
$$

where $k_{1}$ and $k_{2}$ are non-zero constants. The second inequality of (5) implies that

$$
B_{v}^{\mathcal{F}}(1, y)=H^{\mathcal{F}}(y) \cdot \prod_{i=1}^{t}\left(y-a_{i}\right)^{r_{i}-1} ; \quad B_{v}^{\mathcal{L}}(1, y)=H^{\mathcal{L}}(y) \cdot \prod_{i=1}^{t}\left(y-a_{i}\right)^{r_{i}-1},
$$

with $H^{\mathcal{F}}(y)$ and $H^{\mathcal{L}}(y)$ being polynomials of degree $t-1$. Now, since $\mathcal{L}$ is a logarithmic model of $\mathcal{F}$, we have the equality of the Camacho-Sad indices

$$
I_{q_{i}}\left(\pi_{1}^{*} \mathcal{F}, E_{1}\right)=I_{q_{i}}\left(\pi_{1}^{*} \mathcal{L}, E_{1}\right) \quad \text { for } i=1, \ldots, t
$$

Computation of the indices gives that

$$
\begin{gathered}
I_{q_{i}}\left(\pi_{1}^{*} \mathcal{F}, E_{1}\right)=-\operatorname{Res}_{y=a_{i}} \frac{B_{v+1}^{\mathcal{F}}(1, y)}{P_{v+1}^{\mathcal{F}}(1, y)}=-\frac{H^{\mathcal{F}}\left(a_{i}\right)}{k_{1} \cdot \prod_{\substack{j=1 \\
j \neq i}}^{t}\left(a_{i}-a_{j}\right)} \\
I_{q_{i}}\left(\pi_{1}^{*} \mathcal{L}, E_{1}\right)=-\operatorname{Res}_{y=a_{i}} \frac{B_{v+1}^{\mathcal{L}}(1, y)}{P_{v+1}^{\mathcal{L}}(1, y)}=-\frac{H^{\mathcal{L}}\left(a_{i}\right)}{k_{2} \cdot \prod_{\substack{j=1 \\
j \neq i}}^{t}\left(a_{i}-a_{j}\right)}
\end{gathered}
$$

The equalities of the Camacho-Sad indices imply that $H^{\mathcal{F}}(y)=k H^{\mathcal{L}}(y)$ with $k=k_{1} / k_{2}$. Then, we have that $B_{v}^{\mathcal{F}}(1, y)=k B_{v}^{\mathcal{L}}(1, y)$. Moreover, since $P_{v+1}^{\mathcal{F}}(1, y)=k P_{v+1}^{\mathcal{L}}(1, y)$, we get that $A_{v}^{\mathcal{F}}(1, y)=$ $k A_{\mathcal{v}}^{\mathcal{L}}(1, y)$. It follows that $\mathcal{F}$ and $\mathcal{L}$ have the same initial parts $J_{p}(\mathcal{F})$ and $J_{p}(\mathcal{L})$ at $p$.

The same arguments prove the equality $\jmath_{q}(\mathcal{F})=J_{q}(\mathcal{G})$ at each infinitely near point $q$ of $C$, and the result is straightforward.

\section{ACKNOWLEDGMENTS}

The author was partially supported by the research projects MTM2007-66262 (Ministerio de Educación y Ciencia), MTM2006-15338-C02-02 (Ministerio de Educación y Ciencia), VA059A07 (Junta de Castilla y León) and PGIDITI06PXIB377128PR (Xunta de Galicia). 


\section{RESUMO}

Este trabalho fornece uma condição necessária e suficiente a fim de que duas folheações singulares curva generalizada admitam mesma redução de singularidades e mesmo índice de Camacho-Sad em cada ponto infinitamente vizinho.

Palavras-chave: folheações holomorfas singulares, dinâmica complexa.

\section{REFERENCES}

CAMACHO C AND SAD P. 1982. Invariant Varieties Through Singularities of Holomorphic Vector Fields. Ann of Math 115: 579-595.

Camacho C, Lins Neto A And Sad P. 1984. Topological Invariants and Equidesingularisation for Holomorphic Vector Fields. J Differential Geom 20: 143-174.

CORRAL N. 2003. Sur la topologie des courbes polaires de certains feuilletages singuliers. Ann Inst Fourier 53: $787-814$

Rouillé P. 1999. Théorème de Merle: cas des 1-formes de type courbes généralisées. Bol Soc Bras Mat 30: 293-314. 